\title{
Electric Field-Induced Modification of Magnetocrystalline Anisotropy in Transition-metal Films and at Metal-Insulator Interfaces
}

\author{
K. Nakamura ${ }^{1 *}$, T. Akiyama ${ }^{1}$, T. Ito ${ }^{1}$, M. Weinert ${ }^{2}$, and A. J. Freeman ${ }^{3}$ \\ ${ }^{1}$ Department of Physics Engineering, Mie University, Tsu, Mie 514-8507, Japan \\ ${ }^{2}$ Department of Physics, University of Wisconsin-Milwaukee, Milwaukee, Wisconsin 53201, USA \\ ${ }^{3}$ Department of Physics and Astronomy, Northwestern University, Evanston, Illinois 60208 USA
}

(Received 20 February 2011, Received in final form 14 March 2011, Accepted 19 April 2011)

\begin{abstract}
We report results of first principles calculations for effects of an external electric field ( $E$-field) on the magnetocrystalline anisotropy (MCA) in transition-metal (Fe, $\mathrm{Co}$, and $\mathrm{Ni}$ ) monolayers and at metal-insulator (Fe/MgO) interfaces by means of full-potential linearized augmented plane wave method. For the monolayers, the MCA in the Fe monolayer (but not in the Co and Ni) is modified by the $E$-field, and a giant modification is achieved in the $\mathrm{Fe}_{0.75} \mathrm{Co}_{0.25}$. For the $\mathrm{Fe} / \mathrm{MgO}$ interfaces, the ideal $\mathrm{Fe} / \mathrm{MgO}$ interface gives rise to a large out-ofplane MCA, and a MCA modification is induced when an $E$-field is introduced. However, the existence of an interfacial FeO layer between the Fe layer and the MgO substrate may play a key role in demonstrating an $E$ field-driven MCA switching, i.e., from out-of-plane MCA to in-plane MCA.
\end{abstract}

Keywords : magnetocrystalline anisotropy, external electric field, transition-metal films, metal-insulator interfaces, first principles calculations

\section{Introduction}

Magnetism in thin films and at surfaces/interfaces continues to be one of the most studied and important properties of materials, with many exciting discoveries related to both fundamental and applied sciences [1]. Currently, one of key challenges lies in controlling and designing magnetic properties by applying an external electric field $(E-$ field), which provides a new pathway for controlling magnetism at the nano-scale with the promise of ultra-low energy power consumption compared to traditional control using magnetic field [2].

Materials being studied so far include magnetoelectric multiferroics and magnetic semiconductors. Surprisingly, recent experiments demonstrated that even in itinerant transition-metals such as thin films FePt and FePd with liquid interfaces, the coercivity is reversibly varied by application of a voltage [3]. Moreover, the perpendicular magnetocrystalline anisotropy (MCA) energy is significantly reduced by an applied voltage in $\mathrm{Au} / \mathrm{Fe} / \mathrm{MgO}$ junctions, i.e., at $\mathrm{Fe} / \mathrm{MgO}$ interfaces [4], and the direct switching from out-of-plane to in-plane has been achieved in $\mathrm{Au} / \mathrm{Fe}_{0.8} \mathrm{Co}_{0.2} /$

\footnotetext{
*Corresponding author: Tel: +81-59-231-5361

Fax: +81-59-231-9726, e-mail: kohji@phen.mie-u.ac.jp
}

\section{$\mathrm{MgO}$ [5].}

From a theoretical point of view, successive first principles calculations have been performed to determine the magnetoelectric effect in such transition-metal surfaces/ interfaces [6-10], and a general consensus is obtained where the modification of the $d$-electron charge/spin density induced by the $E$-field contributes to the effect. In the present paper, we report first-principle predictions for the $E$-field-induced MCA modification in free-standing transition-metal $(\mathrm{Fe}, \mathrm{Co}$, and $\mathrm{Ni})$ monolayers and metal-insulator $(\mathrm{Fe} / \mathrm{MgO})$ interfaces.

\section{Method}

Calculations were carried out using the full-potential linearized augmented plane-wave (FLAPW) method [11, 12] within the local spin density approximation (LDA) [13] and the scalar relativistic approximation (SRA), using a single slab geometry that allows a natural way to include the $E$-field applied along the surface normal $[7,14]$. To determine the MCA, the second variational method for treating the spin-orbit coupling (SOC) was performed by using the calculated eigenvectors in the SRA, and the MCA energy, $E_{\mathrm{MCA}}$, was determined by the force theorem [15, 16], which is defined as the energy eigenvalue difference for the magnetization oriented along the in-plane [100] and 
out-of-plane [001] directions. 7,056 special k-points in the two-dimensional Brilliouin zone were used to suppress numerical fluctuations in $E_{\mathrm{MCA}}$.

\section{Transition-metal (Fe, Co, Ni) Monolayers}

Table 1 summaries the calculated $E_{\mathrm{MCA}}$ in $E$-fields of zero and $1 \mathrm{~V} / \AA$ for the free-standing $\mathrm{Fe}_{x} \mathrm{Co}_{1-x}(x=1.0,0.75$, 0.5 , and 0.0 ) and $\mathrm{Ni}$ monolayers, where the $\mathrm{Fe}_{0.75} \mathrm{Co}_{0.25}$ and $\mathrm{Fe}_{0.5} \mathrm{Co}_{0.5}$ monolayers are modeled by $2 \times 2$ and $\sqrt{2} \times \sqrt{2}$ ordered alignments, respectively. For the Fe monolayer, the $E_{\mathrm{MCA}}$ in zero field has a positive value indicating out-ofplane MCA, and the $E_{\mathrm{MCA}}$ decreases by $0.17 \mathrm{meV} /$ atom when the $E$-field is introduced. In contrast, for the $\mathrm{Fe}_{x} \mathrm{Co}_{1-x}$ $(x=0.75,0.5,0.0)$ and Ni monolayers, the $E_{\mathrm{MCA}}$ in zero field has negative values of in-plane MCA. When the $E$ field is introduced, the $E_{\mathrm{MCA}}$ for $\mathrm{Fe}_{x} \mathrm{Co}_{1-x}(x=0.75,0.5)$ monolayers increases compared to the Fe monolayer, and a maximum change in the $E_{\mathrm{MCA}}$ by $0.7 \mathrm{meV} /$ atom are obtain-

Table 1. Calculated $E_{\mathrm{MCA}}$ (in meV/unit-area) in electric fields of zero and $1 \mathrm{~V} / \AA$ and their difference, $\Delta E_{\mathrm{MCA}}=E_{\mathrm{MCA}}(1 \mathrm{~V} / \AA)$ $-E_{\mathrm{MCA}}(0 \mathrm{~V} / \AA)$, for $\mathrm{Fe}_{x} \mathrm{Co}_{1-x}(x=1,0.75,0.5$, and 0$)$ and $\mathrm{Ni}$ monolayers. The second column indicates the number of valence electrons.

\begin{tabular}{cccrr}
\hline \hline & \multirow{2}{*}{$N_{\text {valence }}$} & \multicolumn{2}{c}{$E_{\mathrm{MCA}}$} & \multirow{2}{*}{$\Delta E_{\mathrm{MCA}}$} \\
\cline { 3 - 4 } & & Zero field & $1 \mathrm{~V} / \AA$ & \\
\hline $\mathrm{Fe}$ & 8 & 0.19 & 0.02 & -0.17 \\
$\mathrm{Fe}_{0.75} \mathrm{Co}_{0.25}$ & 8.25 & -1.52 & -0.82 & 0.70 \\
$\mathrm{Fe}_{0.5} \mathrm{Co}_{0.5}$ & 8.5 & -1.74 & -1.57 & 0.17 \\
$\mathrm{Co}$ & 9 & -1.37 & -1.39 & -0.02 \\
$\mathrm{Ni}$ & 10 & -1.70 & -1.69 & 0.01 \\
\hline
\end{tabular}
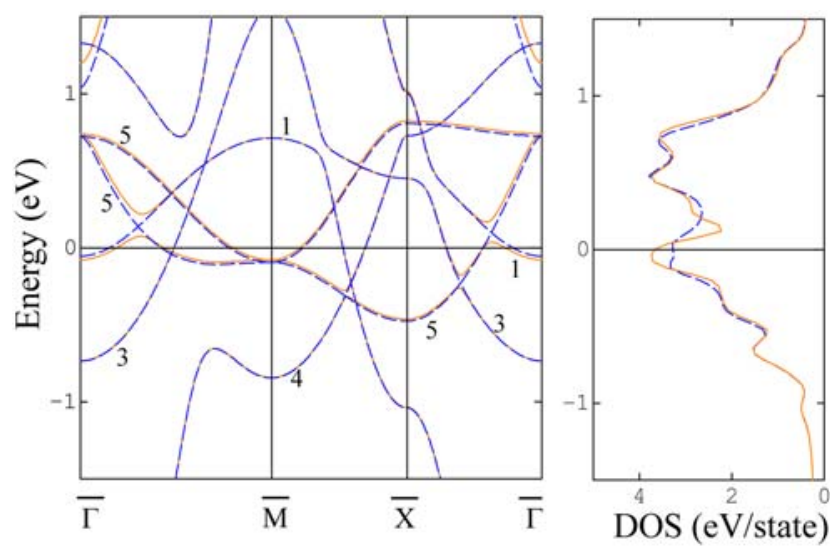

Fig. 1. Calculated minority-spin band structure and density of states (DOS) for an $\mathrm{Fe}(001)$ monolayer in electric fields of zero (dotted lines) and $1 \mathrm{~V} / \AA$ (solid lines). The reference energy $(E=0)$ places the Fermi energy, $E_{\mathrm{F}}$. Bands 1,3 , 4, and 5 stand for $d\left(z^{2}\right), d\left(x^{2}-y^{2}\right), d(x y)$, and $d(x z, y z)$ states, respectively. ed in the $\mathrm{Fe}_{0.75} \mathrm{Co}_{0.25}$ monolayer. In contrast, for the Co and Ni monolayers, the $E_{\mathrm{MCA}}$ do not change with application of the $E$-field.

Fig. 1 shows the minority-spin band structures and density of states (DOS) for the Fe monolayer. In the zero field, the bands crossing the Fermi level $\left(E_{\mathrm{F}}\right)$ arise mainly from the minority-spin states, while the majority-spin bands (not shown in the figure) are almost fully occupied and are located from -1 to $-4 \mathrm{eV}$ below $\mathrm{E}_{\mathrm{F}}$. When the $E$-field is introduced, although the overall the band structure is similar compared to that in zero field, a significant difference is observed near $E_{\mathrm{F}}$, e.g., two intersecting bands 1 and 5 above $E_{\mathrm{F}}$ at $1 / 3(\Gamma-\mathrm{M})$ hybridize with each other and are split by about $0.2 \mathrm{eV}$ by the $E$-field, around $3 / 5(\mathrm{X}-\mathrm{G})$ the lowered band 1 is almost pushed down below $E_{\mathrm{F}}$. These changes in band structures near $E_{\mathrm{F}}$ lead to the MCA modification, since the MCA mainly originates from the SOC between the minority-spin bands crossing $E_{\mathrm{F}}$ as pointed out previously [17].

In addition, when number of valence electrons increases within the rigid band (i.e., $E_{\mathrm{F}}$ shifts up around 0.1-0.2 eV), the large MCA modification may be induced, as observed in the $\mathrm{Fe}_{0.75} \mathrm{Co}_{0.25}$ monolayers mentioned above. In the case of the $\mathrm{Co}$ and $\mathrm{Ni}, E_{\mathrm{F}}$ further shifts above the $d$ bands due to an increase in the number of electrons, and so the states around the induced band gaps become occupied. Hence, no change in the MCA appears in the Co and Ni monolayers.

\section{Metal-insulator (Fe/MgO) Interfaces}

For an ideal abrupt $\mathrm{Fe} / \mathrm{MgO}$ interface, we considered two structures, $\mathrm{Fe} / \mathrm{MgO}(001)$ and $\mathrm{Au}_{3} / \mathrm{Fe}_{3} / \mathrm{MgO}(001)$ structures; the first represents a surface Fe monolayer on an $\mathrm{MgO}(001)$ substrate and the latter, an Au-capped three-atomic-layer $\mathrm{Fe}$ film on an $\mathrm{MgO}$ substrate where the surface magnetoelectric effect $[6,9]$ is eliminated by the nonmagnetic $\mathrm{Au}$ over-layer. The $\mathrm{Fe}$ atoms at the interface are on top of the $\mathrm{O}$ atoms, as found by first-principles calculations [18] and LEED experiments [19]. In order to determine the role of the interfacial $\mathrm{FeO}$ layer, we considered additional structures, $\mathrm{Fe} / \mathrm{FeO} / \mathrm{MgO}$ and $\mathrm{Au}_{3} / \mathrm{Fe}_{3} / \mathrm{FeO} / \mathrm{MgO}$, as observed in experiments [20], where an $\mathrm{FeO}$ layer inserts between the Fe layer and the $\mathrm{MgO}$ substrate. The atomic z-positions are fully optimized using the atomic force FLAPW calculations.

Table 2 gives the calculated $E_{\mathrm{MCA}}$ in zero field and $\pm 1 \mathrm{~V} /$ $\AA$, respectively, where the positive (negative) field is chosen so as to set the positive (negative) electrode at the vacuum region of the $\mathrm{MgO}$ side and the negative (positive) electrode for the $\mathrm{Au}$ sides. In zero field, the $E_{\mathrm{MCA}}$ for the ideal abrupt interface of the $\mathrm{Fe} / \mathrm{MgO}$ and $\mathrm{Au}_{3} / \mathrm{Fe}_{3} / \mathrm{MgO}$ structures have very large positive values of 1.28 and 0.94 $\mathrm{meV}$, respectively, indicating a strong out-of-plane MCA compared to that of the free-standing Fe monolayer. When an $E$-field is introduced, the MCA is modified; for the $\mathrm{Fe} /$ 
Table 2. Calculated $E_{\mathrm{MCA}}$ (in meV/unit-area) in electric fields of zero and $\pm 1 \mathrm{~V} / \AA$ and their difference, $\Delta E_{\mathrm{MCA}}=E_{\mathrm{MCA}}(1 \mathrm{~V} /$ $\AA)-E_{\mathrm{MCA}}(0 \mathrm{~V} / \AA ̊ \AA)$, for $\mathrm{Fe} / \mathrm{MgO}$ interfaces.

\begin{tabular}{lccrr}
\hline \hline & \multicolumn{3}{c}{$E_{\mathrm{MCA}}$} & \multirow{2}{*}{$\Delta E_{\mathrm{MCA}}$} \\
\cline { 2 - 4 } & Zero field & \multicolumn{1}{c}{$-1 \mathrm{~V} / \AA$} & $1 \mathrm{~V} / \AA$ & \\
\hline $\mathrm{Fe} / \mathrm{MgO}$ & 1.28 & 1.43 & 1.18 & -0.10 \\
$\mathrm{Au}_{3} / \mathrm{Fe}_{3} / \mathrm{MgO}$ & 0.94 & 0.89 & 0.98 & 0.04 \\
$\mathrm{Fe} / \mathrm{FeO} / \mathrm{MgO}$ & -0.18 & -0.07 & -0.34 & -0.16 \\
$\mathrm{Au}_{3} / \mathrm{Fe} / \mathrm{FeO} / \mathrm{MgO}$ & -0.56 & -0.44 & -0.80 & -0.24 \\
\hline
\end{tabular}

$\mathrm{MgO}$ structure, a negative $E$-field enhances the MCA by $0.15 \mathrm{meV}$, while a positive one reduces it by $0.10 \mathrm{meV}$. However, for these fields, no magnetization switching from the out-of-plane to the in-plane is realized. Even in the case of the $\mathrm{Au}_{3} / \mathrm{Fe}_{3} / \mathrm{MgO}$ structure, the modification of the MCA is very small, $0.04 \mathrm{meV}$.

In striking contrast, for the $\mathrm{Fe} / \mathrm{FeO} / \mathrm{MgO}$ and $\mathrm{Au}_{3} / \mathrm{Fe}_{3} /$ $\mathrm{FeO} / \mathrm{MgO}$ structures, the $E_{\mathrm{MCA}}$ turns out to have negative values of -0.18 and $-0.56 \mathrm{meV}$, respectively. Thus, the presence of the interfacial $\mathrm{FeO}$ layer drastically changes the MCA. When an $E$-field is introduced, a large MCA modification appears where a negative $E$-field enhances the MCA by about $0.1 \mathrm{meV}$ while a positive one reduces it by about $0.2 \mathrm{meV}$. Based on these results, for demonstrating an $E$-field-driven MCA switching, from out-of-plane MCA to in-plane MCA, the existence of an interfacial $\mathrm{FeO}$ layer may play a key role.

\section{Summary}

We investigated the effects of the $E$-field on the MCA in transition-metal (Fe, $\mathrm{Co}$, and $\mathrm{Ni}$ ) monolayers and at metalinsulator $(\mathrm{Fe} / \mathrm{MgO})$ interfaces, and discussed the $E$-fieldinduced MCA modification. For the monolayers, the MCA in the Fe monolayer (but not in the $\mathrm{Co}$ and $\mathrm{Ni}$ ) is modified by the $E$-field due to the change in the $d$ band structure around $E_{\mathrm{F}}$. For the $\mathrm{Fe} / \mathrm{MgO}$ interfaces, the existence of an interfacial $\mathrm{FeO}$ layer plays a key role in demonstrating an $E$-field-induced MCA modification.

\section{Acknowledgements}

Work at Mie University was supported by a Grant-in-Aid for Scientific Research (No. 20540334) from the Japan Society for the Promotion of Science, and for computations partially performed at ISSP, University of Tokyo. Work at
Northwestern University (DE-FG02-88ER45372) and the University of Wisconsin-Milwaukee (DE-FG02-06ER46328) was supported by the U.S. Department of Energy.

\section{References}

[1] S. D. Bader, Rev. Mod. Phys. 78, 1 (2006), and references therein.

[2] S. D. Bader and S. S. P. Parkin, Annu. Rev. Condens. Matter Phys. 1, 71 (2010), and references therein.

[3] M. Weisheit, S. Fähler, A. Marty, Y. Souche, C. Poinshignon, and D. Givord, Science 315, 349 (2007).

[4] T. Maruyama, K. Ohta, T. Nozaki, T. Shinjo, M. Shiraishi, S. Mizukami, Y. Ando, and Y. Suzuki, Nature Nanotech. 4, 158 (2009).

[5] Y. Shiota, T Maruyama, T. Nozaki, T. Shinjyo, M. Shiraishi, and Y. Suzuki, Appl. Phys. Express 2, 063001 (2009).

[6] C.-G. Duan, J. P. Velev, R. F. Sabirianov, Z. Zhu, J. Chu, S. S. Jaswal, and E. Y. Tsymbal, Phys. Rev. Lett. 101, 137201 (2008).

[7] K. Nakamura, R. Shimabukuro, Y. Fujiwara, T. Akiyama, T. Ito, and A. J. Freeman, Phys. Rev. Lett. 102, 187201 (2009).

[8] M. Tsujikawa and T. Oda, Phys. Rev. Lett. 102, 247203 (2009).

[9] K. Nakamura, R. Shimabukuro, T. Akiyama, T. Ito, and A. J. Freeman, Phys. Rev. B 80, 172402 (2009).

[10] K. Nakamura, T. Akiyama, T. Ito, M. Weinert, and A. J. Freeman, Phys. Rev. B 81, 220409R (2010).

[11] E. Wimmer, H. Krakauer, M. Weinert, and A. J. Freeman, Phys. Rev B 24, 864 (1981).

[12] M. Weinert, E. Wimmer, and A. J. Freeman, Phys. Rev. B. 26, 4571 (1982).

[13] U. von Barth and L. Hedin, J. Phys. C 5, 1629 (1972).

[14] M. Weinert, G. Schneider, R. Podloucky, and J. Redinger, J. Phys.: Condens. Matter 21, 084201 (2009).

[15] M. Weinert, R. E. Watson, and J. W. Davenport, Phys. Rev. B 32, 2115 (1985).

[16] G. H. O. Daalderop, P. J. Kelly, and M. F. H. Schuurmans, Phys. Rev. B 41, 11919 (1990).

[17] D. S. Wang, R. Wu, and A. J. Freeman, Phys. Rev. B 47, 14932 (1993).

[18] C. Li and A. J. Freeman, Phys. Rev. B 43, 780 (1991).

[19] T. Urano and T. Kanaji, J. Phys. Soc. Jpn. 57, 3403 (1988).

[20] H. L. Meyerheim, R. Popescu, N. Jedrecy, M. Vedpathak, M. Sauvage-Simkin, R. Pinchaux, B. Heinrich, and J. Kirschner, Phys. Rev. B 65, 144433 (2002). 